\title{
Retention of time information in forced-choice duration discrimination*:
}

\author{
RAMONA M. CARBOTTE \\ McMaster University, Hamilton, Ontario, Canada
}

\begin{abstract}
We explore the effect on performance in a forced-choice duration-discrimination task of varying the interstimulus interial (ISI) from 0 to $2 \mathrm{sec}$. The durations were brief empty intervals $(115-285 \mathrm{msec})$ bounded by very brief auditory pulses. Performance improved as the ISI increased from 0 to $1 / 2 \mathrm{sec}$, but a further increase in ISI up to 2 sec resulted in little further change in performance. The "time information" derived from a brief interval bounded by auditory pulses does not appear to be susceptible to the very short-term perceptual memory-loss inferred in other auditory discriminations.
\end{abstract}

In many studies concerned with very short-term auditory perceptual memory, a decrement in performance has been obtained as the interstimulus interval (ISI) is increased. In pitch discrimination, a decline in performance is obtained with increasing ISI, provided that a roving standard is used (Harris, 1952; Wickelgren, 1967; Massaro, 1970; Bull \& Cuddy, 1972). The use of a roving standard supposedly precludes the reliance on a long-term memory standard with which to compare each alternative within a trial. Tanner (1962) reported results from a study of amplitude discrimination in which ISI varied from 0 to $10 \mathrm{sec}$. A measure of "efficiency" in performance reached a maximum at $1 / 2 \mathrm{sec}$ separation between the two signals, and then slowly decreased as the ISI was further increased. Kinchla and Smyzer (1967) obtained a monotonic decrease in performance in an amplitude discrimination task where the ISI was varied from 0 to $2 \mathrm{sec}$.

On the other hand, the time information contained in very brief intervals may not be susceptible to the perceptual memory loss which has been inferred from studies like the ones mentioned above. The results of an experiment done by Small and Campbell (1962) suggest that some minimal separation between time intervals may be needed for optimal performance in duration discrimination, after which further increases in ISI up to $3.2 \mathrm{sec}$ do not result in a change in the dependent variable. In this study, Os decided whether the second (variable) member of a pair of filled auditory intervals was longer or shorter than the first (the standard). The time intervals were defined by noise bursts or by pure tones of either 250 or $5,000 \mathrm{~Hz}$. On each trial, the variable interval could have any one of seven durations, ranging from .25 to 2.5 times the standard. The dependent variable was a difference threshold, $\Delta \mathrm{T}_{.5}$ or

*This research was supported by Grant A-7919 from the National Research Council of Canada to A. B. Kristofferson. This report is condensed from a doctoral dissertation submitted to McMaster University in October 1972. The author wishes to express her appreciation to Dr. Kristofferson for many very useful discussions. that $\Delta \mathrm{T}$ required for $50 \%$ "variable longer" judgments. The separations between the intervals were $.05, .2, .8$, and $3.2 \mathrm{sec}$. For a $400-\mathrm{msec}$ standard, $\Delta \mathrm{T}_{.5}$ was the same at all of these ISIs and for the three different types of auditory signals. For $40-\mathrm{msec}$ pure tone standards, $\Delta \mathrm{T}_{.5}$ reached a minimum (asymptotic) value when the ISI was between .2 and $.8 \mathrm{sec}$; but for the $40 \mathrm{msec}$ noise burst standard, $\Delta \mathrm{T}_{.5}$ did not change when the ISI was increased beyond $.2 \mathrm{sec}$.

Data obtained by McKee et al (1970) are consistent with the results summarized above. McKee et al varied the ISI in a forced-choice duration discrimination task, with the intervals being defined by brief visual dark flashes (the time between the offset and onset of a light). They found that varying the ISI from $1 / 2$ to $2 \mathrm{sec}$ had no effect on performance in discriminating between $\mathrm{T}$ and $\mathrm{T}+\Delta \mathrm{T}$; the base duration, $\mathrm{T}$, was $50 \mathrm{msec}$, and $\Delta \mathrm{T}$ was 10 and $30 \mathrm{msec}$. In discussing these results, McKee et al raised the possibility that the ISI variable had no effect because the response might be based on the time information in only one interval; that is, one interval might be consistently ignored on each trial. The $O$ might be maintaining a stable internal standard in long-term memory, with which he can compare either the first or the second interval in the forced-choice situation.

One aim of this experiment was to replicate the results of McKee et al, using empty intervals bounded by brief auditory signals, with the stimulus alternatives chosen in such a way that we could determine whether the $O$ was using both intervals on each trial in the FC task. If changing the separation between the intervals had no effect on $\hat{P}(C)$, it would be highly desirable to rule out the possibility that only one interval was being used on each trial.

A second aim of this experiment was to obtain a sample of single-stimulus (SS) performance in discriminating between $T$ and $T+\Delta T$, after conditions that would seem to be unfavorable to the formation of a stable internal standard, i.e., immediately after the $O$ has had much experience with a FC task involving several standards randomly intermixed. One might 
consider the first interval in the FC task as a standard with which the $O$ can compare the second interval; this standard has to be retained only during the ISI, until the second interval has been presented. This view of the FC situation can be contrasted to the situation in the single-stimulus paradigm where the $\mathrm{O}$ has to compare the single interval presented on each trial with some standard held in long-term memory.

\section{METHOD}

\section{Apparatus and General Procedure}

The $\mathrm{O}$ was seated in a sound-attenuated cubicle isolated from the experimental control room. Auditory signals were presented to the $O$ binaurally over earphones. All events on each trial were controlled by a PDP-8S computer interfaced to an electronic switch (Grason-Stadler), which gated a $2,000-\mathrm{Hz}$ sine wave at zero crossing in its cycle. The rise-decay times of these pulses were set at $1 \mathrm{msec}$, and their duration was programmed for $10 \mathrm{msec}$. The intensity of each of the four pulses defining two time intervals was $.3 \mathrm{rms} \mathrm{V}$. as measured at the switch. The corresponding sound pressure level of a continuous tone, as measured at the earphone, was $72 \mathrm{~dB}$. With no signal being presented, the sound level reading was $54 \mathrm{~dB}$ due to ambient noise from a fan ventilating the cubicle.

On each trial. a 250-msec visual warning signal was followed $2 \mathrm{sec}$ later by a pattern of four brief auditory pulses. The intervals to be discriminated were those between the offset of the first and the onset of the second pulse $\left(T_{1}\right)$, and between the offset of the third and the onset of the fourth pulse $\left(T_{2}\right)$. The interval between Pulses 2 and 3 was designated as the interstimulus interval (ISI). The $\mathrm{O}$ had a forced-choice task; he was instructed to indicate whether $T_{1}$ or $T_{2}$ was the longer interval by pressing one of two microswitches interfaced with the computer. He was told that the longer interval was as likely to be first as second. If a correct response was made within 4 sec of the end of $T_{2}$. feedback was given in the form of two 125 -msec light flashes. The next trial began $1.5 \mathrm{sec}$ later.

On each trial, any one of eight pairs of intervals could occur. These were the pairs

$$
\begin{array}{ll}
\mathrm{T}, \mathrm{T}+\Delta \mathrm{T} & \mathrm{T}+\Delta \mathrm{T}, \mathrm{T} \\
\mathrm{T}, \mathrm{T}-\Delta \mathrm{T} & \mathrm{T}-\Delta \mathrm{T}, \mathrm{T}
\end{array}
$$

for two values of $T: 150$ and $250 \mathrm{msec} . \Delta T$ was constant for each $O$ and was chosen after several practice sessions so as to have a performance level of around .85 in discriminating between 150 and $150+\Delta T$, when the separation between the intervals was $1 \mathrm{sec} . \Delta \mathrm{T}=35 \mathrm{msec}$ was used for two $\mathrm{Os}(\mathrm{V}$ and M.D.) who had taken part in previous experiments on duration discrimination, while $\Delta T=25$ was used for a third, naive $O$.

Each session consisted of four blocks of 80 trials. Within any one session, the ISI was fixed at one of six values: $0.1 / 8,1 / 4$, $1 / 2,1$, or $2 \mathrm{sec}$. The ISI varied from session to session, with each member of the set being used once before the set was cycled through again, in another order. This was repeated four times.

Once within each cycle, a single stimulus session was run. In two sessions, the stimulus alternatives were 150 and $150+\Delta T$, and in another two sessions, the alternatives were 250 and $250+\Delta \mathrm{T}$. The $\mathrm{O}$ was presented with only one interval on each trial, and he had two response alternatives: the interval was to be identified as either "short" or "long." The boundaries of each interval were identical to those in the FC task. Feedback was stimulus dependent, occurring on only those trials on which the interval presented was of magnitude $\mathrm{T}$. Each SS session consisted of three blocks of 100 trials.

\section{RESULTS AND DISCUSSION}

In this experiment, Os were discriminating between the intervals $T_{s}$ and $T_{s}+\Delta T$, where $T_{s}$ (the base duration) had four values: $150-\Delta T, 150,250-\Delta T$, and $250 \mathrm{msec}$. An $S_{1}$ pattern is the ordered pair of intervals $\left(T_{s}+\Delta T, T_{s}\right)$ in which the longer interval occurs first, and an $S_{2}$ pattern is the pair $\left(T_{s}, T_{s}+\Delta T\right)$. $\hat{\mathrm{P}}\left(1 \mid \mathrm{S}_{1}\right)$ is then the proportion of trials on which an $S_{1}$ pattern occurred and the first interval was correctly chosen as the longer one. $\hat{\mathrm{P}}\left(2 \mid \mathrm{S}_{2}\right)$ is defined in a similar way. When $S_{1}$ and $S_{2}$ occur equally often, one measure of the ability to discriminate between $T_{s}$ and $T_{s}+\Delta T$ is given by

$$
\hat{\mathrm{P}}(\mathrm{C})=1 / 2\left[\hat{\mathrm{P}}\left(1 \mid \mathrm{S}_{1}\right)+\hat{\mathrm{P}}\left(2 \mid \mathrm{S}_{2}\right)\right] .
$$

Table 1 lists $\hat{\mathrm{P}}\left(1 \mid \mathrm{S}_{1}\right), \hat{\mathrm{P}}\left(2 \mid \mathrm{S}_{2}\right)$, and $\hat{\mathrm{P}}(\mathrm{C})$ for each $\mathrm{O}$, at each ISI in the FC procedure. The data from the first cycle through the set of ISIs is not included.

\section{Performance as a Function of ISI}

A large improvement in performance occurs as the ISI increases from 0 to $1 / 2 \mathrm{sec}$, but there is relatively little further change in $\hat{\mathrm{P}}(\mathrm{C})$ as the ISI changes from $1 / 2$ to $2 \mathrm{sec}$. Figure 1 shows the effect of ISI upon $\hat{P}(C)$ for the individual Os; the data are averaged over the four base durations. Averaging over base durations, within an $O$, is justified if the processes used at the four base durations are the same, and this may be a reasonable assumption, since all the base durations were intermixed randomly within each session. In Fig. 1 , an increase in $\hat{\mathbf{P}}(\mathrm{C})_{\mathbf{a v}}$ as ISI increases from $1 / 2$ to $1 \mathrm{sec}$ is seen only with one $\mathrm{O}$, as is the decrease in $\mathbf{P}(C)_{a v}$ as ISI goes from 1 to $2 \mathrm{sec}$.

Figure 2 shows the effect of increasing ISI upon $\hat{P}(C)$, for each $\mathrm{O}$, with base duration as the parameter. For each $\mathrm{O}$, the functional relation between $\hat{\mathrm{P}}(\mathrm{C})$ and ISI is determined once at each of four base durations; we shall consider this functional relation to be replicated four times within each $\mathrm{O}$. A decrease in $\hat{\mathrm{P}}(\mathrm{C})$ as ISI increases from 1 to $2 \mathrm{sec}$ is seen in only 3 of the 12 replications shown in Fig. 2. Also, an increase in $\hat{P}(C)$ as ISI goes from $1 / 2$ to $1 \mathrm{sec}$ is seen in only 3 of the 12 replications. Hence, performance reaches an asymptotic value when the interval between the durations to be discriminated is $1 / 2 \mathrm{sec}$, and this level is maintained over the range of ISIs from $1 / 2$ to $2 \mathrm{sec}$.

In general, performance is disrupted when the ISI is made very short. In Fig. 1, all three Os show a decrease in $\dot{P}(C)$ when the ISI is reduced from $1 / 2 \mathrm{sec}$, although for one $O$ there is little difference between performance with adjacent intervals (ISI $=0$ ) and with intervals separated by $2 \mathrm{sec}$. On the other hand, in the individual data shown in Fig. 2 there seems to be no consistent pattern in the shape of the functional relation between $P(C)$ and ISI over the range of $0-1,2 \mathrm{sec}$. There may be a 
Table 1

Results from FC Procedure With ISI Varied from 0 to 2 Sec [Each $P(C)$ is Based on 240 Trials]

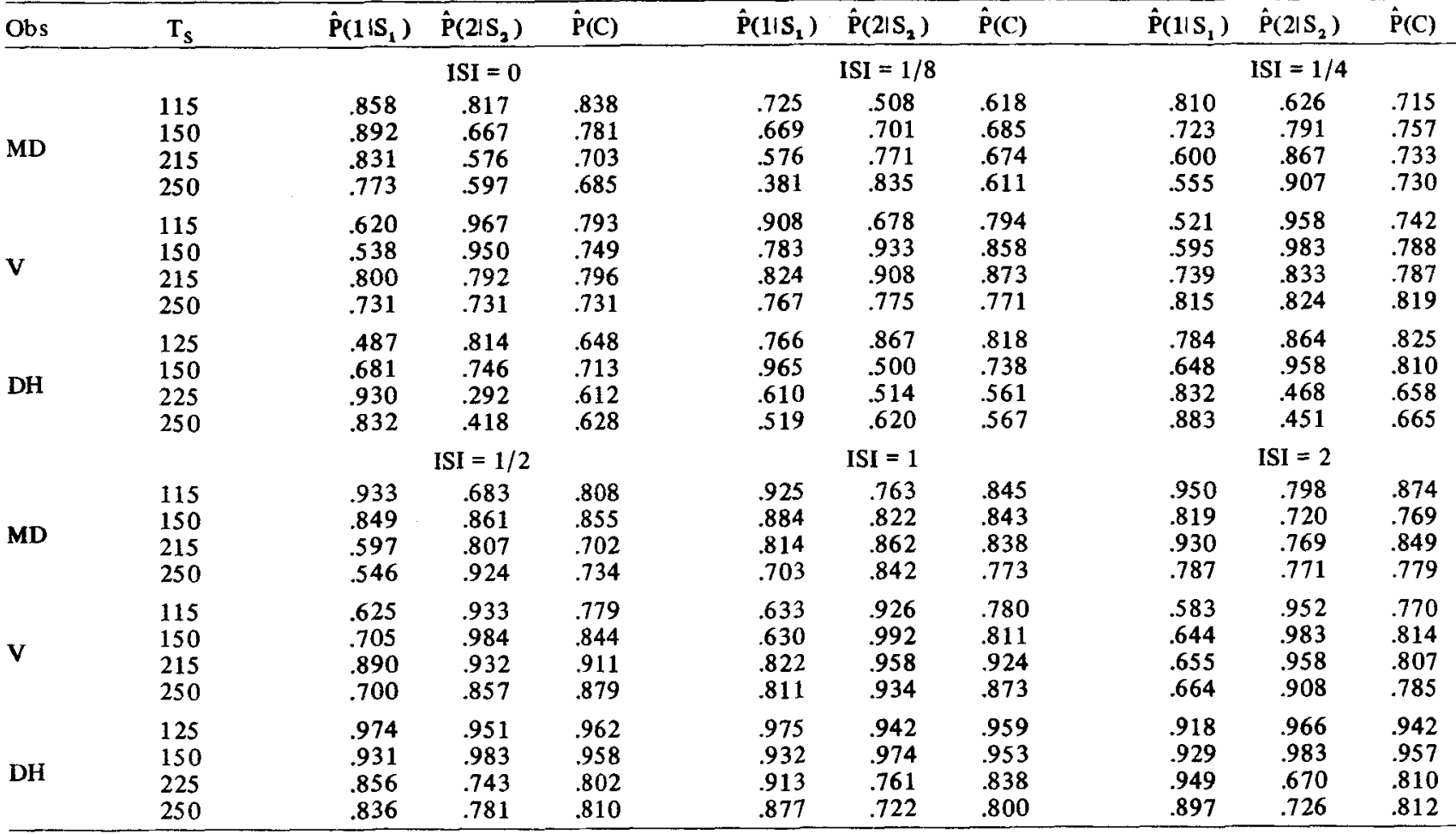

variety of ways in which an $\mathrm{O}$ can deal with very short ISIs.

\section{Are Both Intervals Used?}

The observed independence of $\hat{P}(C)$ and ISI when the

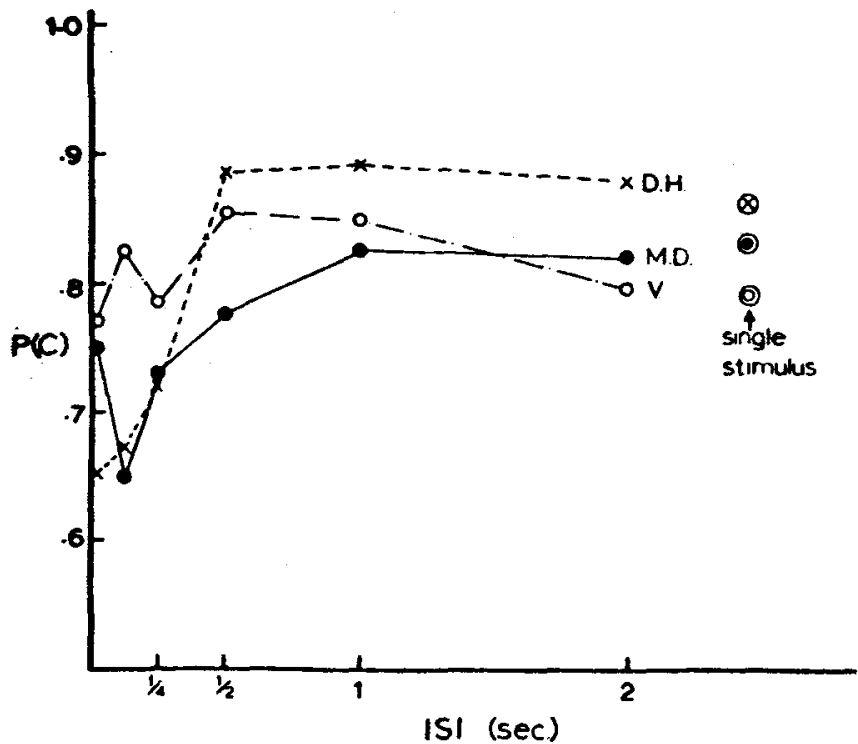

Fig. 1. Effect of ISI upon $\hat{\mathbf{P}}(\mathrm{C})$, for individual Os; data averaged over four base durations. The circled data points represent performance from sessions with the single stimulus procedure, averaged over two base durations.

latter exceeds $.5 \mathrm{sec}$ is not due to the O's use of only one interval on each trial. This conclusion is based on the

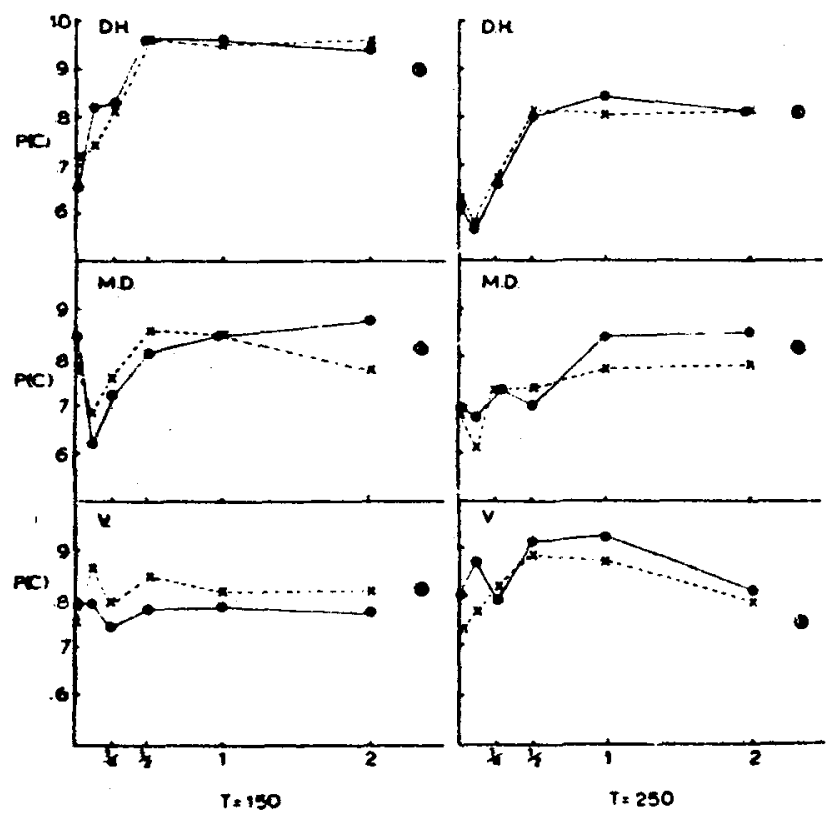

Fig. 2. The effect of ISI upon $\hat{P}(C)$, at each of four base durations. Individual data. The panel on the right shows $\hat{\mathbf{P}}(\mathrm{C})$ at base durations $(250-\Delta \mathrm{T}), \bullet-\bullet$, and $250, x \cdots \times$. The panel on the left shows $P(C)$ at base durations $(150-\Delta T)$, $\bullet$, and $150, x \cdots \cdots \times$. The circled data points show $\hat{\mathbf{P}}(C)$ from sessions with the SS procedure. 
Table 2

Single Stimulus Performance [Each $P(C)$ is Based on 600 Trials]

\begin{tabular}{lcccc}
\hline Obs & $\mathrm{T}$ & $\hat{\mathrm{P}}($ short $\mathrm{T})$ & $\hat{\mathrm{P}}($ long $\mathrm{T}+\Delta \mathrm{T})$ & $\hat{\mathrm{P}}(\mathrm{C})$ \\
\hline $\mathrm{MD}$ & 150 & .85 & .83 & .84 \\
$(\Delta \mathrm{T}=35)$ & 250 & .80 & .82 & .81 \\
$\mathrm{~V}$ & 150 & .80 & .85 & .82 \\
$(\Delta \mathrm{T}=35)$ & 250 & .72 & .77 & .74 \\
$\mathrm{DH}$ & 150 & .88 & .92 & .90 \\
$(\Delta \mathrm{T}=25)$ & 250 & .82 & .81 & .81 \\
\hline
\end{tabular}

following considerations. Suppose that on some proportion $(\phi)$ of the trials, the information from only the first interval is used in making the decision as to which is the longer interval, on some proportion ( $\psi$ ) only the second interval is used, and on each of the remaining trials both trials are used. Let the various possible pairs of intervals be those summarized in (I). where $T$ can be either longer or shorter than the interval accompanying it on a given trial. On those trials where the $O$ uses only the first interval and $T$ occurs first, the probability of a correct response will be at chance, since the $O$ does not have the essential information from the second interval. On the other hand, when the $O$ uses only the first interval and $T$ occurs second, the proportion of correct responses will be $P_{s s}$, the probability of correctly discriminating between $T+\Delta T$ and $T-\Delta T$ in what is now essentially a single stimulus situation. Let $\mathbf{P}\left(\mathrm{P}^{\prime}\right)$ represent the probability of a correct response when $T$ occurs first (second) and the $O$ uses both intervals on a trial. The following matrix summarizes the probabilities of a correct response, conditional on the "processing strategy" used on a particular trial and on whether T occurs first or second in the stimulus pattern.

\section{Processing strategy: $O$ uses}

first only second only both intervals

$\begin{array}{lccc}\text { T first } & .5 & \mathrm{P}_{\text {ss }} & \mathrm{P} \\ \text { T second } & \mathrm{P}_{\text {ss }} & .5 & \mathrm{P}^{\prime}\end{array}$

Setting $\epsilon=1-\phi-\Psi$, we can write

$$
\mathrm{P}(\mathrm{C} \mid \mathrm{T} \text { first })=\phi(.5)+\Psi \mathrm{P}_{\mathrm{ss}}+\epsilon \mathrm{P}
$$

and

$$
\mathrm{P}(\mathrm{C} \mid \mathrm{T} \text { second })=\phi \mathrm{P}_{\mathrm{ss}}+\Psi(.5)+\epsilon \mathrm{P}^{\prime}
$$

When $\mathrm{T}$ occurs first or second equally often, we can combine Eqs. 1 and 2 to obtain

$$
\mathrm{P}(\mathrm{C} \mid \mathrm{T})=1 / 2\left[(\phi+\Psi)\left(\mathrm{P}_{s s}+.5\right)+\epsilon\left(\mathrm{P}+\mathrm{P}^{\prime}\right)\right]
$$

From this last expression, we can easily obtain a lower bound on $\epsilon$ (by setting $\mathrm{P}_{\mathrm{ss}}, \mathrm{P}$, and $\mathrm{P}^{\prime}$ equal to 1 , which is their maximum possible value, and then solving for $\epsilon$ ):

$$
\varepsilon \geqslant \frac{\mathrm{P}(\mathrm{C} \mid \mathrm{T})-.75}{.25}
$$

In this experiment, we can calculate the quantities

$$
\hat{\mathbf{P}}(\mathrm{C} \text { T first })=1 / 2[\hat{\mathrm{P}}(1 \quad \mathrm{~T} . \mathrm{T}-\Delta \mathrm{T})+\hat{\mathrm{P}}(2 \mid \mathrm{T}, \mathrm{T}+\Delta \mathrm{T})]
$$

and

$$
\hat{\mathrm{P}}(\mathrm{C} ; \mathrm{T} \text { second })=1 / 2[\hat{\mathrm{P}}(2: \mathrm{T}-\Delta \mathrm{T}, \mathrm{T})+\hat{\mathrm{P}}(1 \mid \mathrm{T}+\Delta \mathrm{T}, \mathrm{T})]
$$

for two values of $T$. For ISIs greater than $1 / 4 \mathrm{sec}$, these are above chance level, so we can reject the possibility that only the first, or only the second interval, is being used throughout the experiment. Moreover, for each $\mathrm{O}$, $\dot{\mathrm{P}}(\mathrm{C} \mid \mathrm{T})$ is greater than .75 for at least one value of $\mathrm{T}$, with ISIs of $0,1 / 2,1$, and $2 \mathrm{sec}$. Hence, according to Inequality 3 . the decision as to which is the longer interval is being based on both intervals on some nonzero proportion of the trials. ${ }^{\text {t }}$

\section{Relating FC and SS Performance}

Table 2 summarizes SS performance. $\hat{\mathrm{P}}($ short $\mid \mathrm{T})$ is the proportion of trials on which $T$ was presented and the response was "short." $\hat{\mathrm{P}}$ (long | $\mathrm{T}+\Delta \mathrm{T})$ is defined in a similar way. All the data collected under this procedure are included, even those from the initial blocks of trials. The circled data points in Figs. 1 and 2 show $\hat{\mathbf{P}}(C)$ with the SS task; in this case, $P(C)$ is defined by

$$
\hat{\mathrm{P}}(\mathrm{C})=1 / 2[\hat{\mathrm{P}}(\text { short } \mid \mathrm{T})+\hat{\mathrm{P}}(\text { long } \mid \mathrm{T}+\Delta \mathrm{T})]
$$

In the individual data shown in Fig. 2, there is no tendency for the discriminability of $T$ and $T+\Delta T$ to be consistently better in the FC situation (with ISI $=2 \mathrm{sec}$ ) than in the SS situation.

Even if the time information from a single interval were coded in the same way in the FC and SS situations, a difference in performance might be predicted, depending on the decision strategy assumed for the FC task. For example, if the measures of the intervals $T$ and $T+\Delta T$ were represented by normally distributed random variables with equal variance, and a choice of the longer interval in the FC task were made on the basis of the difference between these measures, then the "signal detectability" measure, d", from the FC task should be larger than that from the SS task, by a factor of $\sqrt{2}$ (Green \& Swets, 1966). In Creelman's (1962) model, the variances are unequal, and the discrepancy between FC and SS performance will depend on the magnitude of both $T$ and $\Delta T$. Hence, the similarity in the FC and SS performance levels seen in this experiment suggests that if the measure of an interval $T$ can be represented by a 
continuous random variable whose variance is independent of $T$, then the choice of the longer interval in the FC task is not being based on a difference between the measures of the two intervals.

Alternatively, we could assume that after each interval is presented, a decision is made as to whether the interval is "long" or "short" compared to some criterion, and that the choice of the longer interval in a pair is made according to the combined outcome of the two decisions. With this decision strategy for the FC task, the "onset-offset delay" model of Allan. Kristofferson, and Wiens (1971) predicts that the sensitivity measure $d_{\mathbf{q}}$ will be the same in the FC and SS situations (see Kristofferson \& Allan, 1973). An equality would be predicted for the performance measure P(C) only if the criterion were the same in the two tasks. ${ }^{2}$

It would be of considerable interest to use these data to test the available quantitative models for duration discrimination, but that is beyond the scope of this paper. If we have an adequate model of the processes involved in the discrimination of very brief time intervals, we should be able to relate performance in the FC and SS conditions. That is, with the data from one task, we should be able to obtain estimates of parameters of the model, which we can then use to predict performance in the other situation.

\section{SUMMARY AND CONCLUSIONS}

Our finding that performance in a FC duration discrimination task does not deteriorate as the ISI increases from $1 / 2$ to $2 \mathrm{sec}$ has also been obtained with empty time intervals defined by visual dark flashes of the order of $50 \mathrm{msec}$ (McKee et al, 1970) and is consistent with results obtained with filled auditory durations of the order of 40 and $400 \mathrm{msec}$ (Small \& Campbell, 1962). ${ }^{3}$ Whatever information is being retained during the ISI, it is not susceptible to the same kind of decay over a few seconds as is often seen in certain other auditory discriminations in which two observations are to be compared. It may be that the time information in an interval is not susceptible to very short-term sensory memory loss. Or it may be that a decision about the first interval is compared or combined with a decision about the second interval, and this decision is more durable than the sensory information in a stimulus. The assumption that a decision is made after each interval seems plausible in the light of the disruption in performance which occurs with very short ISIs. Moreover, an equality of FC and SS performance can be predicted by certain quantitative models for duration discrimination if we assume that a decision occurs after each interval, and we have found that the performance levels from the two tasks are very close in this experiment.

\section{REFERENCES}

Allan, L. G., Kristofferson, A. B., \& Wiens, E. W. Duration discrimination of brief light flashes. Perception \& Psychophysics, 1971, 9, 327-334.

Bull, A. R., \& Cuddy, L. L. Recognition memory for pitch of fixed and roving stimulus tones. Perception \& Psychophysics, $1972,11,105-109$.

Creelman, C. D. Human discrimination of auditory duration. Journal of the Acoustical Society of America, 1962, 34, 582-593.

Green, D. M., \& Swets, J. A. Signal detection theory and psychophysics. New York: Wiley, 1966.

Harris, J. D. The decline of pitch discrimination with time. Journal of Experimental Psychology, 1952, 43, 96-99.

Kinchla, R. A., \& Smyzer, F. A. A diffusion model of perceptual memory. Perception \& Psychophysics, 1967, 2, 219-229.

Kristofferson, A. B., \& Allan, L. G. Successiveness and duration discrimination. In S. Komblum (Ed.), Attention and performance IV. New York: A cademic Press, 1973.

Massaro, D. W. Forgetting: Interference or decay? Journal of Experimental Psychology, 1970, 83, 238-243.

McKee, M. E., Allan, L. G., \& Kristofferson, A. B. Duration discrimination of brief visual off-flashes. Technical Report No. 42, Department of Psychology, McMaster University, 1970.

Small, A. M., \& Campbell, R. A. Temporal differential sensitivity for auditory stimuli. American Journal of Psychology, 1962 , $75,401-410$.

Tanner, W. P. Physiological implications of psychophysical data. Annals of the New York Academy of Sciences, 1961, 89, 752-765.

Wickelgren, W. A. Consolidation and retroactive interference in short-term recognition memory for pitch. Journal of Experimental Psychology, 1967, 72, 250-259.

\section{NOTES}

1. The inequality (3) suggests a form of "catch trial" for the forced choice procedure: By including a set of stimulus alternatives as in (I), with $\Delta T$ large enough so that $\dot{P}(C \mid T)=1$, we should be able to obtain a lower bound on $\epsilon$ which approaches 1 .

2. Creelman's model would make a similar prediction, if this decision strategy were assumed.

3. In this experiment, the ISI at which we obtained symptotic values for performance was independent of the size of the standard or base duration, while this was not the case in the Small and Campbell study. Filled intervals of the order of 40 and $400 \mathrm{msec}$ may well be qualitatively different, whereas empty intervals in the range of $115-285 \mathrm{msec}$ may be dealt with in the same way, especially if there is trial-to-trial uncertainty as to which base duration will occur.

(Received for publication January 26, 1973; accepted June 13,1973.) 